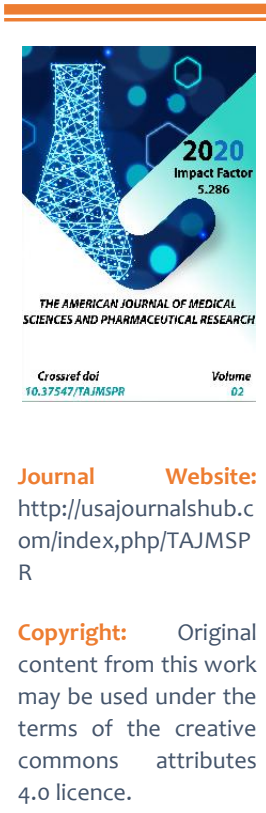

\title{
Dental Status In Geriatric Population
}

\author{
Samatov Ulugbek Abdumajitovich \\ Chair of hospital and clinic stomatology Andijan State Medical Institute, Uzbekistan
}

\section{ABSTRACT}

Biological phenomenon. As a result of the advances made in medicine and public health measures in the last half of the 2oth century, there is a substantial increase in the life span of man. Elders above 65 years (old age) have health problems as a result of aging process, which calls for special consideration.

\section{KEYWORDS}

Dental status, geriatric population?preventive dentistry components

\section{INTRODUCTION}

During the latter half of the 2oth century, the age composition of the population changed dramatically, with more people living to older ages and the older population getting older. This demographic change will have a major impact on the delivery of general and oralhealth care, as well as on the providers of these services. Although some older adults have physical and/or psychological conditions that require special attention in the dental office setting, one should not assume that all older people share these conditions.[1,2]

According to the $\mathrm{WHO}$, the global population is increasing at the annual rate of $1.7 \%$, while the population of those over 65 years is increasing at a rate of $2.5 \%$. Both the developed, as well as the lesser-developed 
countries, are expected to experience significant shifts in the age distribution of the population by 2050 . The fastest growing population segment in most countries is the adults older than 80 years, which according to the United Nations estimates will make up nearly $20 \%$ of the world's population.

In India, with its population of over one billion people, people older than 60 years constitute $7.6 \%$ of the total population, which amounts to 76 million. Incidence of oral cancer, which is an old age disease, is highest in India.[3]

Of added concern may be the presence of systemic disease that not only influences the patient's ability to maintain oral hygiene and promotion of oral health, but can actually be related to the occurrence of certain oral diseases. Though impairments are not life threatening, they affect a person's quality-oflife. Thus, planning treatment for the senior dental patient includes an understanding of the chronic diseases the patient lives with daily, as this play a critical role in the acceptance and success of the dental treatment plans.

\section{The "elderly" segment of the population}

People aged 65-74 years are the new or young elderly who tend to be relatively healthy and active;

People aged 75-84 years are the old or midold, who vary from those being healthy and active to those managing an array of chronic diseases;

People 85 years and older are the oldest-old, who tend to be physically frailer. This last group is the fastest-growing segment of the older adult population.

There is a sudden blast of the "65 plus" population in the last decade, and India is no exception to that.[4]

Oral Health Status in Aged

Nutrition in old age and its implications for oral care
Adequate nutrition is a vital factor in promoting the health and wellbeing of the aged. Inadequate nutrition may contribute to an accelerated physical and mental degeneration. Poor oral health can be a detrimental factor to nutritional status and health. Disorders of the oral cavity have contributed to poor eating habits in the elderly. Loose painful teeth or ill-fitting dentures may result in a reduced desire or ability to eat. A compromised nutritional status, in turn can further undermine the integrity of the oral cavity are closely interrelated, diet and nutrition should be considered as an integral part of the oral health assessment and management of the elderly.

Caloric requirements usually decrease in the elderly because of a decline in the basal metabolic rate, brought on by reduced lean muscle mass and lower exercise levels. Appetite and food intake may also decrease, leading to an insufficient caloric intake and frequently results in insufficient consumption of calcium, iron and zinc more frequently in females. Approximately $8000 \mathrm{~kJ}(1900 \mathrm{kcal})$ is the required calorie requirement in 80 years old. An active elderly subject requires a protein intake of $0.97 \mathrm{~g} / \mathrm{kg}$ of body weight per day. However patients suffering from tissues necrosis or inflammation shows an increase in protein turnover and requirements. Among the vitamins, most nutrients are recommended in the same amounts for elderly as for younger people. However, certain groups of elderly, such as those homebound, with no access to sunlight, may have insufficient vitamin $D$ and develop osteomalacia. The other important nutrients required by the older individuals are ascorbic acid, iron, and potassium.[5]

Dental status is considered to be an important contributing factor to health and adequate 
nutrition in elderly. Missing dentition and illfitting dentures cause difficulty in chewing and perception of taste of foods.

The dentists are hence in an ideal position to contribute to the well-being of the elderly population. Dentists should be alert to nutritional risk factors in the elderly population and by careful screening can intervene in the early stages of nutritional problems when such interventions can be most valuable and effective.

\section{Changes in salivary glands and salivary secretion with aging}

The diminished function of salivary gland is commonly associated with aging. The implications of disordered salivary gland maintenance of oral health. The presence of saliva protects the oral cavity the upper airway and digestive tract and facilitates numerous sensorimotor phenomena. The absence of saliva thus has many deleterious consequences to the host.

With advancing age, there is an atrophy of acinar tissue, a proliferation of ductal elements and some degenerative changes in the major salivary glands. These alterations tend to occur linearly with increasing age. Minor salivary glands also undergo similar degenerative changes with advancing age. Thus, there is a normal, uniform decrease in the acinar content of salivary gland tissue accompanying the aging process.[6]

However, it is difficult to make a general conclusion about age-related status of fluid output from salivary glands. It appears that decreased salivary flow does not uniformly accompany the aging in healthy persons. These functional observations contrast with morphologic changes seen in aging salivary glands. One explanation that has been hypothesized to account for this is that salivary glands possess a functional reserve capacity, enabling the glands to maintain a constant fluid output throughout the human adult life span.[6]

The main oral health problems of old age that is mouth dryness and dental caries have been attributed to the reduced salivary flow.

Age changes in oral mucous membrane The oral mucosa performs essential protective functions that profoundly affect the general health and well-being of the host.

A decline in protective barrier function of the oral mucosa could expose the aging host to myriads of pathogens and chemicals that enter the oral cavity during daily activities.

Both histologic layers of the oral mucosa, the epithelium, and connective tissue, have important defensive functions. A stratified epithelium, containing closely apposed, attached cells, and constitutes a physical barrier that interferes with the entry of toxic substances and microorganism. Mucosal epithelial cells also synthesize several substances that are critical for maintenance of the mucosal surface, such as keratin and laminin.[7]

Oral mucosal surfaces also possess a protective self-cleansing mechanism provided by the natural turnover of the epithelial cells.

Earlier studies report that the oral mucosa becomes increasingly thin, smooth with age and that it acquires satin like edematous appearance with loss of elasticity and stippling. The tongue in particular is reported to show marked clinical changes and to become smoother with loss of filiform papillae. With age, there is a tendency for development of sublingual varices and an increasing susceptibility to various pathological conditions such as Candidal infctions and a decreased rate of wound healing.[8]

An additional complication in evaluating oral mucosal status in older persons is the use of 
prosthetic appliances, which have considerable potential to alter mucosal integrity if not maintained properly.

\section{Changes in the teeth with aging}

The gradual changes taking place in the dental tissues after the teeth are fully formed are referred to as age changes. Most of the tissues have a physiological turnover of their components but however, some tissues do not exhibit any turnover such as the enamel. The macroscopic changes taking place with age in the teeth change in form and occur with age. Wear and attrition affect the tooth form. The perikymata and imbrication lines are lost, giving the enamel surface a flat appearance with less detail than in newly erupted teeth.

The altered surface structure gives the teeth in older individuals a different pattern of light reflection, which causes a change in the observed color. Changes in the dentin, both in quantity (thickness) and quality also result in a gradual loss of transparency. Pigmentation of anatomical defects, corrosion products and inadequate oral hygiene may also change the tooth color. 8

All the changes in enamel are based on ionexchange mechanisms. It becomes less permeable and possibly more brittle with age. The nitrogen content of enamel is showed to increase with age. No explanation could be offered to account for the increase in organic material, but probably the filling in of the cracks by organic material (acquired lamellae).

A two age dependent change takes place in dentin:

Continued growth, referred to as physiological secondary dentin formation.

Gradual obturation of the dentinal tubules referred to as dentin sclerosis.

The dental pulp in teeth from old individuals differs from that in younger teeth by having more fibers and fewer cells, and hence reduces in volume.

The blood supply, including the rich plexus of capillary loops in the subodontogenic region, is greatly reduced. These changes are important because the pulp cannot be expected to have the same reparative capacity as the younger teeth. Electron microscopy of old pulps has shown loss and degeneration of both myelinated and unmyelinated nerves and thus affected the healing capacity of pulp. Pulp calcifications are also found to increase in frequency, number and size with age. Diffuse calcification and narrowing of the root canals with increasing age.

Cementum apparently continues to be laid throughout life, but the rate of formation diminishes with age. Under some circumstances, excess amounts of cementum may be formed (hypercementosis) associated with accelerated elongation of an unopposed tooth or to an inflammatory stimulus.

Furthermore increase in the fluoride and magnesium content is seen with age. The cementum may contain one of the very few biomarkers of age. Countable, microscopically clear annular rings have been found in teeth that might aid in age determination in forensic specimens.

Age changes in morphology of teeth have important clinical implications as these changes may influence the outcomes of the restorative treatments and also have a great bearing on the reparative responses.

\section{Aging and periodontal disease}

Globally, the percentage of the subjects with community periodontal index scores 4 (deep pockets) ranges from approximately 5-70\% among older people.[9]Periodontal diseases are among the most prevalent chronic conditions in dentate older populations. Several epidemiological surveys have found 
that the prevalence and severity of periodontal diseases increase with age.

Periodontal disease in the elderly does not appear to be specific disease but the result of a chronic adult periodontitis since adulthood although age-related changes have been documented in the periodontium of elders, these changes do not appear to be the cause of periodontal disease in the elderly. Enhanced severity of periodontal diseases with age has been related to the length of time the periodontal tissues have been exposed to the dentogingival bacterial plaque and is considered to reflect the individual's cumulative oral history. However, the susceptibility of the periodontium to plaqueinduced periodontal breakdown may be influenced by the aging process or by a specific health problems of the aging patient.[10]

At the biological level, aging is associated with changes that lead to a progressive, irreversible deterioration of the functional capacities of several tissues and organs. Changes in structure and function during aging may affect the host response to plaque microorganisms and may influence the rate of periodontal destruction in older people. The greater amount of plaque recovered in the elderly subjects could be due, in part, to a larger area for plaque retention because of the gingival recession. Further, exposed cementum of the root surface and dental enamel constitute two unlike types of hard dental tissues with distinct surface characteristics, which may influence the plaque formation rate differently. Differences in dietary habits, increased flow of gingival exudate from the inflamed gingiva and possible age-related changes in salivary gland secretions may similarly alter the conditions for growth and multiplication of the plaque microorganisms.

\section{Problems of providing preventive dental care for elderly people}

One of the major challenges in providing restorative as well as preventive care for elderly people is to develop an appreciation of the need for regular care.[11] Globally, poor oral health among older people has particularly been seen in a high level of tooth loss, dental caries experience, high prevalence rates of periodontal disease, xerostomia, and oral precancer/cancer.[12] The basis of prevention is related to detecting disease at the earliest possible stage, which requires regular patient contact. The many factors that are known to influence older people's utilization of dental services directly or indirectly can be divided into four main categories.

- Illness and health related factors

- Oral health status.

- Experiencing discomfort.

- General ill health.

- Mobility, functional limitation.

- Socio-demographic factors

- Place of residence.

- Education.

- Income.

- Age.

- Sex.

- Culture.

- Ethnicity.

- Service-related factors

- Accessibility.

- Dentist behavior.

- Dentist attitude.

- Price of service

- Satisfaction with service.

- Transport.

- Attitudinal or subjective factors.

- Personal beliefs.

- Feeling no need, perceived need.

- Perceived importance. 
- Fear and anxiety.

- Resistance to change.

- Perceived financial strain.

- Satisfaction with dental visits.

- Preventive Dentistry Components

- Mechanical plaque removal

Several reports worldwide have shown that use of professional dental health services is low among older people, particularly among the socio-economically disadvantaged.[13] The preferred method of brushing for most elders is sulcular brushing with soft toothbrush (Bass method). Persons with gingival recession should be instructed to observe certain precautions avoid further recession or cemental abrasion. These may include the use of an extra soft toothbrush, use of light pressure, modification of the brushing method. The plaque retention in the elderly is exacerbated by the presence of restorations, missing teeth and gingival recession. The wearing of removable dentures may also negatively influence plaque accumulation. In addition, they often face difficulty in mechanical removal of plaque because of reduced manual dexterity or impaired vision or due to physical limitations associated with conditions such as stroke, Parkinson's disease or severe arthritis.

The elderly person should be helped to develop the ability to brush effectively and thoroughly. Those who have diminished manual dexterity may benefit from the use of traditional mechanical toothbrushes, rotary electric toothbrushes, or manual brushes that have been adapted or customized for each person.

\section{CONCLUSION}

The major block in oral health care of elderly and the residents would be the underestimation of the oral health care need by them. The dental care of the residents is often limited to emergency care and is not aimed at retaining teeth. Conversely, with changing attitudes the oral health goal should include: Keeping their teeth, keeping their teeth healthy and keeping their teeth pretty. The best option to serve the residents would be "home dentistry or domiciliary dental care," however it is yet an infrequent practice in India. Surveys should be conducted in this sector very routinely to spot the residents in the need of oral care circumscribing nursing homes, old age homes, ashrams, secure units, and community households.

\section{REFERENCES}

1. Harris NO. 6th ed. New York: Prentice Hill; 1999. Primary Preventive Dentistry. [Google Scholar]

2. Park K. 21st ed. Jabalpur: Bhanot Publishers; 2011. Preventive and Social Medicine. [Google Scholar]

3. Panchbhai AS. Oral health care needs in the dependant elderly in India. Indian J Palliat Care. 2012;18(1):19-26. [PMC free article] [PubMed] [Google Scholar]

4. National Programme for the Health Care of the Elderly (NPHCE) Directorate General of Health Services Ministry of Health \&Family Welfare Government of India [Google Scholar]

5. Soini $H$, Routasalo $P$, Lauri $S$, Ainamo A. Oral and nutritional status in frail elderly. Spec Care Dentist. 2003;23:209-15. [PubMed] [Google Scholar]

6. Vissink A, Spijkervet FK, Amerongen VA. Aging and saliva: Areview of the literature. Spec Care Dentist. 1996;16(3):95-103. [PubMed] [Google Scholar] 
7. Holm-Pedersen P, Loe H. 2nd ed. London: Wiley; 1997. Textbook of Geriatric Dentistry. [Google Scholar]

8. Papas AS, Niessen LC, Chauncey HH. St. Louis: Mosby Yearbook; 1991. Geriatric Dentistry -Aging and Oral Health. [Google Scholar]

9. World Health Organization. WHO Oral Health Country/Area Profile. [Last accessed on 2014 Oct 03]. Available from: http://www.whocollab.od.mah.se/index.h tml.

10. Suresh R. Prevention and Treatment of age Related Diseases. The Netherlands: Springer; 2006. Aging and periodontal disease; pp. 193-200. [Google Scholar]

11. Slaughter A. Providing Dental Care for Older Adults in Long Term Care. University of Pennsylvania School of Medicine. 2006 [Google Scholar]

12. Schou L. Oral health, oral health care and oral health promotion among older adults: Social and behavioural dimensions. In: Cohen LK, Gift HC, editors. Disease Prevention and Oral Health Promotion. Copenhagen: Munksgaard; 1995. [Google Scholar]

13. Petersen PE, Holst D. Utilization of dental health services. In: Cohen L, Gift HC, editors. Disease Prevention and Oral Health Promotion. Copenhagen: Munksgaard; 1995. [Google Scholar] 